\title{
Large-eddy simulations of the flow and acoustic fields of a rocket jet impinging on a perforated plate
}

\author{
Mathieu Varé* and Christophe Bogey ${ }^{\dagger}$ \\ Univ Lyon, École Centrale de Lyon, INSA Lyon, Université Claude Bernard Lyon I, CNRS \\ Laboratoire de Mécanique des Fluides et d'Acoustique, UMR 5509 \\ F-69134 Ecully, France
}

\begin{abstract}
Large-eddy simulations (LES) of five overexpanded rocket jets impinging on a plate located at a distance of $30 r_{0}$, where $r_{0}=D / 2$ is the nozzle radius, and of the corresponding free jet have been performed. The jets are at an exhaust Mach number of 3.1 and a Reynolds number of $2 \times 10^{5}$. Four plates are perforated with a hole of diameter $h=1.33 D, 2 D, 3 D$ and $4 D$, whereas the last one is not, in order to investigate the effects of the hole on the jet flow and acoustic fields. The acoustic levels are highest for the non-perforated plate, and decrease as the hole diameter increases, due to weaker interactions between the jet and the plate. In comparison with the levels of the free jet, they are higher by about $5 \mathrm{~dB}$ for the full plate, $4 \mathrm{~dB}$ for $h=1.33 D$ and $2 D, 3 \mathrm{~dB}$ for $h=3 D$ and $2 \mathrm{~dB}$ for $h=4 D$. In the upstream direction, for the free jet, the broadband shock associated noise (BBSAN) is dominant. For the impinging jets, spatial Fourier transforms and two-dimensional spatial correlations show that the main upstream noise component is produced by the impingement of the jet turbulent structures on the plate, and that the reflections of the Mach waves on the plate are negligible. In the downstream direction, for the free jet and down to the plate for the impinging jets, the acoustic field is dominated by Mach waves produced by the convection of the jet coherent structures at a supersonic velocity. Downstream of the plate, sound waves are generated by interactions between the jet flow and the plate.
\end{abstract}

\section{Introduction}

During a rocket launch, the hot supersonic gases of the engines are canalized in a trench dug under the rocket. However, a part of the jets impinges on the ground, which generates intense acoustic waves. Those waves propagate upstream to the fairing, where they might excite the rocket structure and damage the payload. Thus, the understanding of noise generation at the lift-off of a space launcher is a main concern for the aerospace industry.

In order to analyze noise generation during a rocket launch, a simplified geometry of a launch pad, namely a jet impinging on a plate with a hole, can be considered. Such a configuration has been investigated numerically for hot overexpanded supersonic jets, typical of rocket jets, for nozzle-to-plate distances $L$ between $15 D$ and $20 D$, where $D=2 r_{0}$ is the nozzle diameter [1-3]. In particular, Kawai et al. [1] studied the impingement of an overexpanded jet at an exit Mach number of 3.66 on a plate with a hole using an axisymmetric Large-Eddy Simulation (LES). They observed a strong acoustic radiation in the upstream direction, which they identified as the reflections of the jet Mach waves on the plate. For a similar geometry, Tsutsumi et al. [2] simulated a jet at an exit Mach number of 3.7. They highlighted the presence of another significant noise component in the upstream direction, generated by the impingement of the jet turbulent structures on the hole edges. This component was also visualized in the acoustic far field of a jet at a Mach number of 3.1 impinging on a perforated plate computed by Troyes et al. [3]. Nevertheless, it is still unclear which of the two upstream components, the impingement noise and the reflections of the Mach waves, dominates. Moreover, the acoustic levels depend on two geometrical parameters, namely the nozzle-to-plate distance $L$ and the hole diameter $h$.

The effects of the nozzle-to-plate distance have been investigated in the simulations of a rocket launch by Tsutsumi et al. [4]. These authors considered a rocket with five jets impinging on a plate with five holes aligned with the jets and four nozzle-to-plate distances of $6 D, 11 D, 16 D$ and $21 D$. A maximum of the pressure levels near the nozzles is found for $L=16 D$. As for the influence of the hole, it has been examined by Tsutsumi et al. [2] for jets at a Mach number of 3.7. In their study, a free jet, a jet impinging on a flat plate and three other ones impinging on a plate with a hole of diameter $h=2 D, 3 D$ or $4 D$ were computed. For all impinging jets, the nozzle-to-plate distance was equal to $20 D$. The

\footnotetext{
*Postdoctoral fellow, mathieu.vare@ec-lyon.fr

†CNRS Research Scientist, Associate Fellow, christophe.bogey@ec-lyon.fr
} 
overall sound pressure levels were found to decrease when the hole diameter increases, with a reduction varying from 2 $\mathrm{dB}$ for $h=2 D$ up to $4 \mathrm{~dB}$ for $h=4 D$ compared with the flat-plate configuration. In other studies on impinging jets at a Mach number lower than 2 [5- 8 , intense tones were also shown to be generated by feedback loops establishing between the nozzle exit and the plate. Such tones do not appear to emerge for Mach numbers around 3, suggesting there is no feedback loops or only weak ones in that case.

In the present work, six overexpanded supersonic jets at an exit Mach number $M_{e}$ of 3.1 and a Reynolds number $R e_{D}$ of $2 \times 10^{5}$ are simulated by LES. One jet is free, and the five other ones impinge on a plate located at a distance $L=30 r_{0}$ from the nozzle exit. Four of the plates have a hole of diameters $h=1.33 D, 2 D, 3 D$ and $4 D$, whereas the fifth one has no hole. The first objective in this study is to investigate the effects of the hole and its diameter on the sound radiated in the upstream direction. For that purpose, the jet flow and acoustic fields are described. In particular, the spectra of the pressure fluctuations in the vicinity of the nozzle are examined. The second objective is to identify the components of the acoustic radiation upstream but also downstream of the plate. To this end, a two-dimensional spatial Fourier transform is applied to the acoustic pressure fields in order to highlight the main propagation directions of the sound waves. Two-dimensional space-time correlations are also used to reveal the propagation of the acoustic waves produced on the plate.

This paper is organized as follows. The jet parameters and numerical methods used in the LES are documented in section [II The results of the simulations are presented in section III] Finally, concluding remarks are given in section IV]

\section{Parameters}

\section{A. Jet parameters}

The parameters of the simulated jets are provided in table 1 The jets have an exhaust Mach number $M_{e}=u_{e} / c_{e}$ of 3.1 and a Reynolds number $R e_{D}=u_{e} D / v_{e}$ of $2 \times 10^{5}$, where $u_{e}$ is the exhaust velocity, $c_{e}$ is the sound celerity and $v_{e}$ is the kinematic viscosity at the jet nozzle exit. The exhaust temperature $T_{e}$ is $738 \mathrm{~K}$ and the exhaust pressure $p_{e}$ is $0.63 p_{0}$, where $p_{0}=10^{5} \mathrm{~Pa}$ is the ambient pressure. The parameters of the corresponding ideally expanded jet are given in table 1. In particular, the ideally expanded Mach number is equal to $M_{j}=2.9$. The ejection parameters of the jets have been chosen to match those of a mixed hydrogen-air jet considered in some experiments conducted at the MARTEL facility [9] and in the simulations of Troyes et al. [3]. The jet static temperature $T_{e}$ is set so that the ratio $c_{e} / c_{0}$ between the local and the ambient sound speeds is the same as that in the hydrogen-air jet, following the approach of Doty \& McLaughlin [10].

\begin{tabular}{cccccc}
\hline$M_{e}$ & $p_{e} / p_{0}$ & $T_{e} / T_{0}$ & $M_{j}$ & $T_{j} / T_{0}$ & $D_{j} / D$ \\
\hline 3.1 & 0.63 & 2.5 & 2.9 & 2.2 & 0.9
\end{tabular}

Table 1 Jets parameters: exit Mach number $M_{e}$, pressure $p_{e}$ and temperature $T_{e}$ and ideally expanded Mach number $M_{j}$, temperature $T_{j}$ and diameter $D_{j}$.

The first jet, labelled as jetfree, is free. The second one, jeth0, impinges on a full plate. The four other ones, jeth1, jeth2, jeth3 and jeth4, impinge on a plate with a hole of diameter $h=1.33 D, 2 D, 3 D$ and $4 D$, respectively. For the impinging jets, the nozzle-to-plate distance $L$ is equal to $30 r_{0}$. The width $e$ of the plates with a hole is arbitrarily chosen equal to $r_{0}$. The nozzle-to-plate distance and the two hole diameters $h=1.33 D$ and $2 D$ are the same as in the MARTEL experiments [9].

The six jets exhaust from a cylindrical nozzle of length $2 r_{0}$, at the inlet of which Blasius boundary layer profiles with a thickness $\delta$ of $0.15 r_{0}$ are imposed. Vortex rings non-correlated in the azimuthal direction are added in the boundary layer at $z=-r_{0}$ to trigger the boundary layer transition from a fully laminar to a disturbed state [11]. The radial profiles of mean velocity and root-mean-squared values of the axial velocity fluctuations thus obtained at the nozzle exit are plotted in figure 1 For the mean velocity, in figure 11(a), the profiles are very similar for the six jets. They differ from the boundary layer profiles imposed at the nozzle inlet. They decrease slowly down to $\left\langle u_{z}\right\rangle=0.93 u_{e}$ at $r=0.8 r_{0}$, then they are drastically reduced below to zero at $r=0.9 r_{0}$. Therefore, near the nozzle exit, the boundary layer is slightly detached from the wall due to the overexpansion of the jets [12], explaining the discrepancies between the nozzle-exit and the nozzle-inlet profiles. For the axial turbulent intensity, in figure 1 b), for all jets, the radial profiles reach a peak value at $r \approx 0.9 r_{0}$. The peak intensities are between $1 \%$ and $1.5 \%$ of the exit velocity, indicating weakly disturbed 
nozzle-exit conditions.

(a)

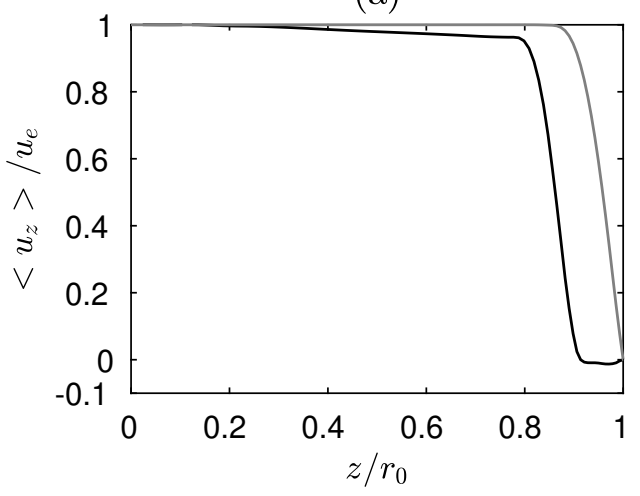

(b)

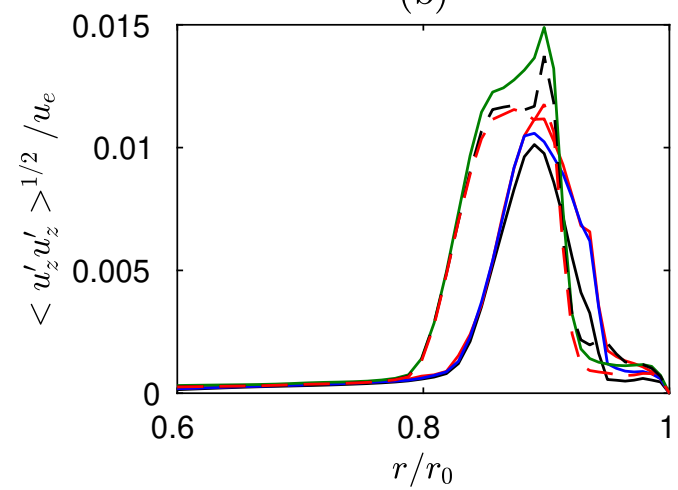

Fig. 1 Radial profiles of (a) mean axial velocity $\left\langle u_{z}\right\rangle / u_{e}$ at - the nozzle-exit and - at the nozzle-inlet and (b) axial turbulence intensity $\left\langle u_{z}^{\prime} u_{z}^{\prime}\right\rangle^{1 / 2} / u_{e}$ at the nozzle-exit: - jeth0, — jeth1, — jeth2, - - - jeth3, - - - jeth4 and - jetfree.

\section{B. Numerical methods}

The numerical set-up is identical to that used in recent LES of subsonic and supersonic, free [13, 14] and impinging [8, 15] jets. In the simulations, the unsteady compressible Navier-Stokes equations are solved in cylindrical coordinates $(r, \theta, z)$ using an OpenMP based in-house solver. The time integration is performed using a six-stage Runge-Kutta algorithm and the spatial derivatives are evaluated with eleven-point low-dispersion finite-difference schemes [16]. At the end of each time step, a selective filtering is applied to remove grid-to-grid oscillations [17]. This filter dissipates kinetic turbulent energy near the grid cut-off frequency, thus acting as a subgrid-scale model [18]. Solid and adiabatic wall conditions are implemented at the plate and nozzle walls. In order to handle shock waves, a damping procedure using a dilatation-based shock detector and a second-order filter are used to remove Gibbs oscillations in the vicinity of shocks [17]. The radiation boundary conditions of Tam \& Dong [19] are imposed to the radial and lateral boundaries of the computational domain. They are used in combination with sponge zones using grid stretching and Laplacian filtering to prevent significant spurious reflections [20]. The method of Mohseni \& Colonius [21] is applied to remove the singularity on the jet axis. The first point close to the axis is located at $r=\Delta r / 2$, where $\Delta r$ is the radial mesh size. The effective azimuthal resolution near the origin of the polar coordinates is reduced down to $2 \pi / 16$ to increase the time step of the simulation [22].

\section{Computational parameters}

The number of points of the mesh grids used in the simulations are provided in table 2 In the six simulations, they are equal to 501 and 256 in the radial and azimuthal directions, respectively. In the axial direction, the numbers of points are equal to 2628 for jetfree, 1910 for jeth0 and 2950 for the jets impinging on perforated plates. The grids thus contain between 250 and 380 millions of points. They extend out to $r=15 r_{0}$ in the radial direction. In the axial direction, they extend down to $z=30 r_{0}$ in the case with no hole and down to $z=50 r_{0}$ in the other cases. The variations of the radial mesh spacing are presented in figure 2(a). It is equal to $\Delta r=0.025 r_{0}$ on the axis and progressively decreases down to $\Delta r=0.0072 r_{0}$ in the shear layer at $r=r_{0}$. Farther from the jet axis, it then increases to reach $\Delta r=0.05 r_{0}$ at $r=5 r_{0}$, which allows us to obtain a Strouhal cut-off number $S t=f D / u_{e}$ of 1.62 for an acoustic wave discretized with 5 points per wavelength, where $f$ is the frequency. The variations of the axial mesh spacing are plotted in figure $2(\mathrm{~b})$. It has a minimum value of $\Delta z=0.014 r_{0}$ at the nozzle exit. For the free jet, it increases up to $\Delta z=0.03 r_{0}$ at $z=50 r_{0}$. For the impinging jets, the axial mesh spacing grows up to $\Delta z=0.022 r_{0}$ at $z=20 r_{0}$, and then is constant. For $z \geq 25 r_{0}$, it is reduced down to its minimum value on the plate at $z=30 r_{0}$. For the plates with a hole, the axial mesh size increases downstream of the plate up to $\Delta z=0.03 r_{0}$ at $z=50 r_{0}$. The extrema values of the mesh spacings and the elongation rates in radial and axial directions are the same as those in the simulations of jets at a Mach number of $M=2$ of Pineau $\&$ Bogey [23, 24]. The results presented in this work are obtained for a simulation time of $1000 r_{0} / u_{e}$. 


\begin{tabular}{ccccc}
\hline & $N_{r}$ & $N_{\theta}$ & $N_{z}$ & $N_{r} \times N_{\theta} \times N_{z}$ \\
\hline jetfree & 501 & 256 & 2628 & $3.4 \times 10^{8}$ \\
jeth0 & 501 & 256 & 1910 & $2.5 \times 10^{8}$ \\
jeth1, jeth2, jeth3, jeth4 & 501 & 256 & 2950 & $3.8 \times 10^{8}$
\end{tabular}

Table 2 Mesh parameters: numbers of points $N_{r}, N_{\theta}$ and $N_{z}$ in the radial, azimuthal and axial directions, and total numbers of points.

(a)

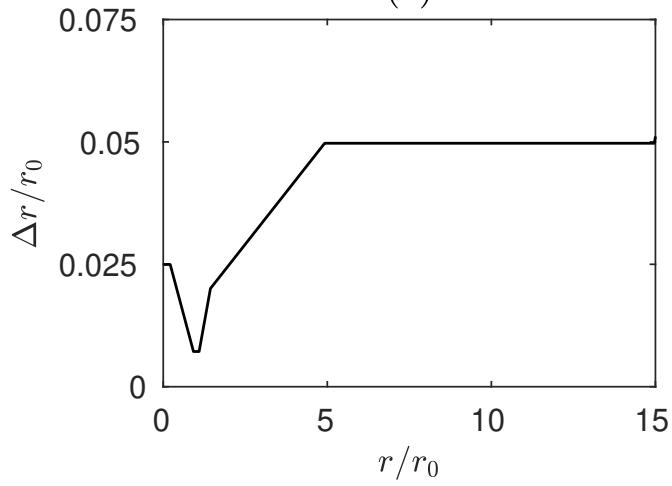

(b)

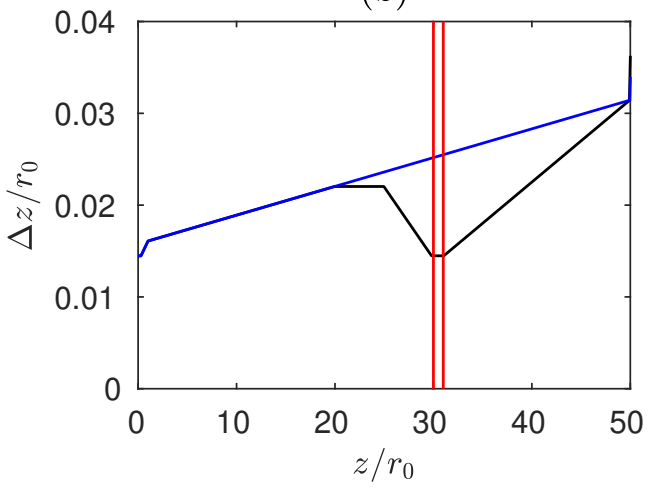

Fig. 2 Variations of (a) radial and (b) axial mesh spacings: — impinging jets, — free jet, — positions of the upstream and downstream faces of the plate.

\section{Results}

\section{A. Snapshots of the flow and acoustic fields}

Snapshots of temperature and pressure fluctuations inside and outside of the flow, respectively, are provided in figure 3. For the six jets, diamond patterns characteristic of shock cells are visible in the jet flows downstream of the nozzle exit. The cells are progressively weakened by the turbulent mixing for $z \geq 10 r_{0}$. For jeth0, jeth 1 and jeth2, in figures $3(\mathrm{a}-\mathrm{c})$, a wall jet is created by the impingement of the flow on the plate. The wall jet is most developed for jeth0 for the plate with no hole and is less apparent for jeth1 and jeth2. For these three jets, zones of high temperature are found near the center of the plate and near the hole edges in the impingement area. For jeth 3 and jeth4, on the contrary, the jets pass through the plate, interacting weakly with the hole edges.

In the pressure fields of figures 3 (a-e), spherical acoustic waves can be observed for the impinging jets, centered on the impingement zone. They are particularly visible for $z \leq 5 r_{0}$. Their levels are highest for jeth 0 and they seem to decrease as the hole diameter increases. For $z \geq 5 r_{0}$, inclined wavefronts of strong amplitude are also seen to propagate in the downstream direction. They are typical of Mach wave radiation, as noticed in several previous simulations of free jets at Mach numbers higher than 2 [25-28]. These waves are produced by the convection of turbulent coherent structures at a supersonic speed. The Mach angle $\alpha$ between the direction of propagation of the Mach waves and the jet axis can be evaluated with the relation

$$
\alpha=\cos ^{-1}\left(\frac{c_{0}}{u_{c}}\right)
$$

where $u_{c}$ is the mean convection velocity of the turbulent structures of the jet. This velocity has been estimated using velocity cross-correlations in the mixing layers between $z=10 r_{0}$ and $20 r_{0}$ and it is close to $u_{c}=0.55 u_{e}$ for all jets, yielding $\alpha=68^{\circ}$ using equation (11). This value is consistent with the inclination of the Mach waves in figure 3 For the plates with a hole, in figures 3 (b-e), the sound field downstream of the plate has no clear organization, even if acoustic waves seem to originate from the hole. In addition, no oblique wavefronts are present, indicating that no Mach waves are generated for $z \geq L$. Finally, for the free jet, in figure 3(f), Mach waves propagate in the downstream direction. 
(a)

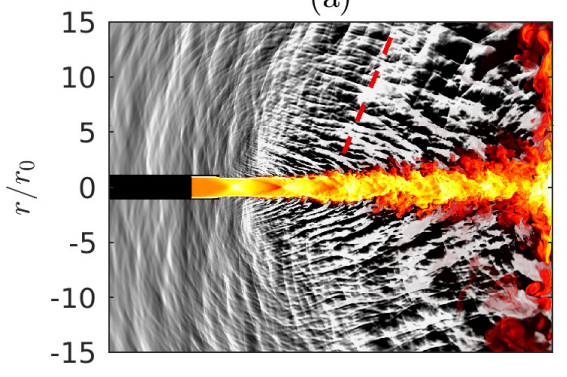

(b)

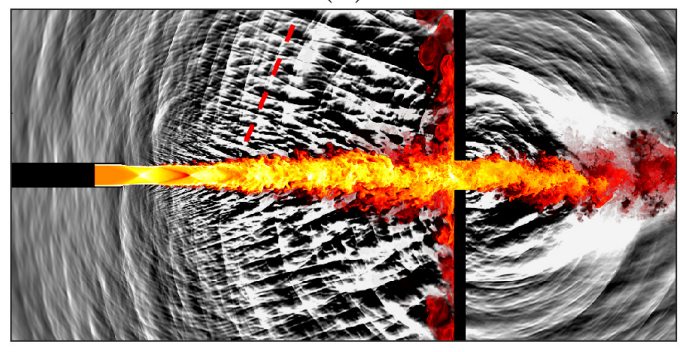

(d)

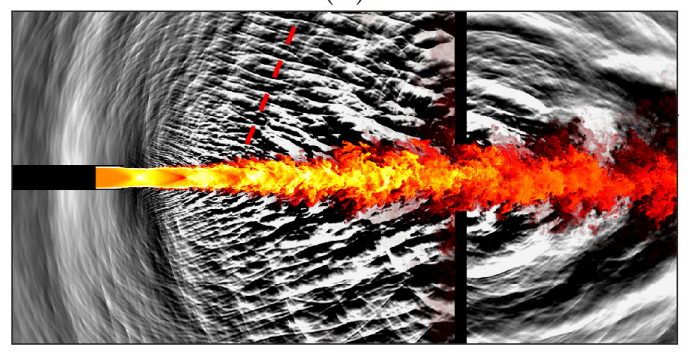

(f)

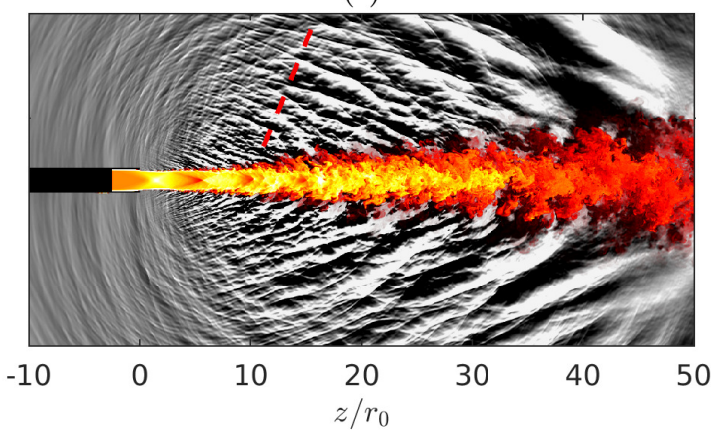

Fig. 3 Snapshots in the $(z, r)$ plane of temperature fluctuations in the flow and of pressure fluctuations outside for (a) jeth0, (b) jeth1, (c) jeth2, (d) jeth3, (e) jeth4 and (f) jetfree. The color scales range from 0 to $780 \mathrm{~K}$ for temperature from red to white and from -2000 to $2000 \mathrm{~Pa}$ for pressure from black to white. The red dashed line indicates the angle of $\alpha=68^{\circ}$ with respect to the jet axis. 
Sound waves of weak intensity are also emitted upstream. This upstream radiation corresponds to the broadband shock associated noise (BBSAN) produced by the interactions between the turbulent structures of the mixing layers and the shock cells.

\section{B. Mean flow fields}

The variations of the jet centerline mean axial velocity are presented in figure 4 a). The results obtained are similar for all jets down to $z=28 r_{0}$, indicating a weak influence of the plate. Significant velocity oscillations are visible for $z \leq 18 r_{0}$. They are linked to the presence of six shock cells, which are progressively damped by the turbulent mixing. The mean value of the length $L_{s}$ of the first four cells is close to $4.6 r_{0}$, in agreement with the experiments of Piantanida \& Berterretche [9] and the simulations of Troyes et al. [3, 29] and Langenais et al. [26]. In order to estimate the shock cell length, Tam \& Tanna [30] proposed the following formula based on the work of Pack [31]

$$
L_{s}=\frac{\pi D_{j} \beta}{\mu_{1}}
$$

where $\beta=\sqrt{M_{j}^{2}-1}$ and $\mu_{1}=2.40483$ is the first zero of the zero-order Bessel function of the first kind. For the present jets, the equation (2) yields a cell length $L_{s}=6.4 r_{0}$, which is larger than the values from the simulations. This may be due to the fact that equation (2) applies to weak shock cells [32], with $\left|M_{e}^{2}-M_{j}^{2}\right| \leq 1$. Indeed, for the jets in this work, $\left|M_{e}^{2}-M_{j}^{2}\right|$ is equal to 1.2 . Furthermore, the mean axial velocity oscillates around the exhaust velocity down to $z \approx 20 r_{0}$. More precisely, the end of the potential core, defined by the position where the centerline axial velocity is equal to $0.9 u_{e}$, is found at $z_{c}=15.7 r_{0}$. This potential core length can be compared with that predicted with the empirical formula proposed by Tam et al. [32]

$$
\frac{z_{c}}{D_{j}}=4.2+1.1 M_{j}^{2}+\left\{\exp \left[-3.2\left(\frac{T_{j}}{T_{0}}-1\right)\right]-1\right\}
$$

yielding $z_{c}=23 r_{0}$ for the present jets. Therefore, the potential core is shorter in the LES, which can be explained by the fact that the constants in relation (3) are based on measurements performed for a jet at a Mach number of 2.2 [33]. Downstream of the potential core, for jeth0, the mean axial velocity is drastically reduced down to zero at $z=30 r_{0}$ on the plate. For the other jets, for $z \geq 30 r_{0}$, the velocity decreases more gradually. Small differences are however noted between the jets. The sonic core, in which the axial velocity is higher than $c_{e}$, closes around $z=40 r_{0}$. More precisely, its length is equal to $38.4 r_{0}$ for jetfree, $42.4 r_{0}$ for jeth1, $41.4 r_{0}$ for jeth $2,42.6 r_{0}$ for jeth 3 and $39.5 r_{0}$ for jeth 4 , revealing no clear relation between the length of the sonic core and the hole diameter. For the jets impinging on a plate with a hole, the sonic core closes after the plate, in agreement with the simulations of Troyes et al. [3].

The jet shear-layer momentum thickness $\delta_{\theta}$ obtained for the different jets is represented in figure $4(\mathrm{~b})$. Downstream of the nozzle, it increases and reaches a value of $\delta_{\theta}=0.56 r_{0}$ at $z \approx 28 r_{0}$, which is close to the value of $0.65 r_{0}$ obtained by Langenais et al. [26] for a similar free jet. Oscillations due to the shock cells are also visible down to $z=28 r_{0}$. For jetfree, for $z \geq 28 r_{0}$, the shear-layer thickness grows roughly linearly. For jeth0, it increases up to a maximum of $0.69 r_{0}$ at $z=29.5 r_{0}$ because of the wall jet. For the other impinging jets, for $z \geq 28 r_{0}$, it decreases down to its minimal value at $z=L$, then it grows again downstream of the plate. For $z \geq L$, it is lowest for $h=1.33 D$ and increases with the hole diameter. The spreading rate of the shear layers is higher for the impinging jets than for the free jet, which leads to shear layers at $z=50 r_{0}$ slightly thicker for jeth3 and jeth4 than for jetfree.

The rms values of the axial velocity fluctuations at $r=r_{0}$ for the free and impinging jets are shown in figure $4(\mathrm{c})$. From the nozzle exit down to $z=2.5 r_{0}$, they are very low. Then, they grow sharply up to a peak value of $0.18 u_{e}$ at $z \approx 11 r_{0}$. The location and the amplitude of the peak are close to those of the simulation of a free jet at $M_{e}=3.3$ by De Cacqueray et al. [25], for which a peak of axial turbulent intensity of $0.21 u_{e}$ is found at $z=12 r_{0}$. For jeth0, for $z \geq 28 r_{0}$, the amplitude of the fluctuations falls down to zero on the plate. For the other jets, the axial turbulent velocity decreases more slowly. For $z \geq 30 r_{0}$, the turbulent levels are similar, with differences around $2 \%$ of the jet exit velocity between the different cases.

\section{Overall sound pressure levels}

The overall sound pressure levels (OASPL) obtained at $r=15 r_{0}$ and at $z=0$ are plotted in figure 5 . The sound levels calculated at $r=15 r_{0}$ are displayed in figure $5 \mathrm{a}$ (a). For the free jet, they increase with the axial distance up to a maximum value of $160 \mathrm{~dB}$ at $z=29 r_{0}$. Then, they slowly decrease down to $155 \mathrm{~dB}$ at $z=50 r_{0}$. For jeth0, the OASPL 
(a)

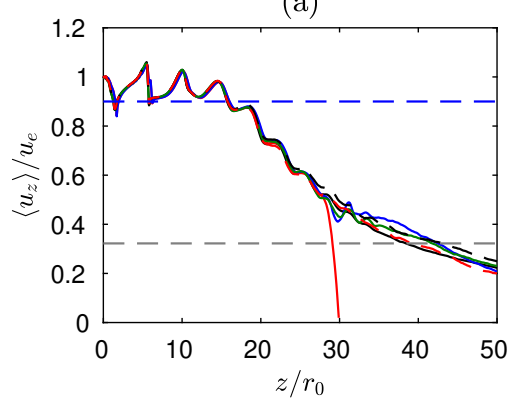

(b)

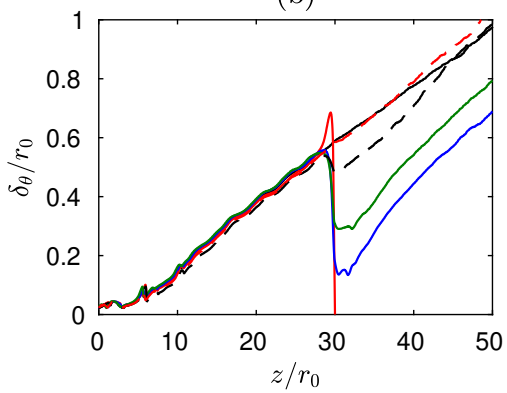

(c)

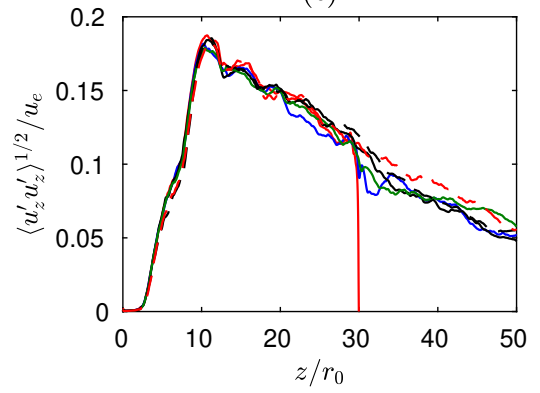

Fig. 4 Variations of (a) the mean axial centerline velocity $\left\langle u_{z}\right\rangle / u_{e}$, (b) the shear-layer momentum thickness $\delta_{\theta} / r_{0}$ and (c) the axial turbulence intensity $\left\langle u_{z}^{\prime} u_{z}^{\prime}\right\rangle^{1 / 2} / u_{e}$ at $r=r_{0}$ for — jetfree, — jeth0, 一 jeth1, - jeth2, - - jeth3 and - - - jeth4; - - $\left\langle u_{z}\right\rangle=0.9 u_{j}$ and $---\left\langle u_{z}\right\rangle=c_{e}$.

also first increase with the axial distance and reach a peak value of $168 \mathrm{~dB}$ at $z=28 r_{0}$. The peak strongly emerges because hydrodynamic pressure fluctuations of the wall jet are present in this near-plate region. Outside of this region, the OASPL of jeth0 are higher by between 3 and $7 \mathrm{~dB}$ than those of the free jet. For the jets impinging on a plate with a hole, the OASPL upstream of the plate vary as those for jeth0. However, they decrease with the hole diameter. In comparison with the case with no hole, the acoustic levels are lower by $0.5-1 \mathrm{~dB}$ for jeth1 and jeth2, 2-3 dB for jeth3 and 2-4 dB for jeth4. This may be caused by weaker interactions between the jet and the plate, as the hole is larger. Downstream of the plate, the sound levels for the impinging jets are lower than for the free jet, which can be explained by the shielding of the jet acoustic radiation by the plate. This hypothesis is also supported by the fact that the levels are the lowest for the plate with the smallest hole and that they grow with the hole diameter.

The OASPL obtained at $z=0$ are represented in figure 5 (b) in order to focus on the noise radiated upstream. For jetfree and jeth4, they increase with the radial distance, by $1.5 \mathrm{~dB}$ between $r=2 r_{0}$ and $r=15 r_{0}$, whereas for the other jets, they do not vary much. The levels are highest for jeth0, with a value of $152.5 \mathrm{~dB}$, and they decrease as the hole diameter increases. Compared with jeth0, they are reduced by $0.7 \mathrm{~dB}$ for jeth1, $1.7 \mathrm{~dB}$ for jeth2, $2.7 \mathrm{~dB}$ for jeth3, $4.7 \mathrm{~dB}$ for jeth4 and $7.7 \mathrm{~dB}$ for jetfree.

(a)

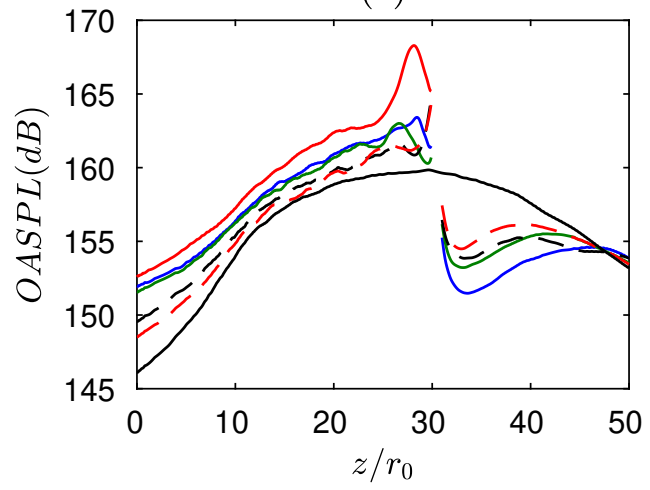

(b)

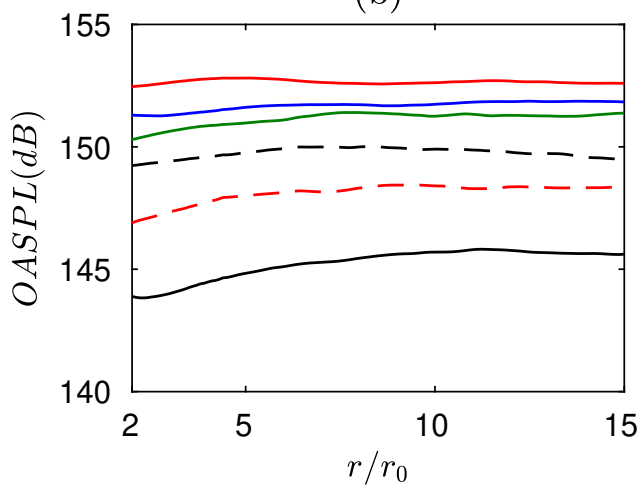

Fig. 5 Overall sound pressure levels at (a) $r=15 r_{0}$ and (b) $z=0$ for — jetfree, — jeth0, — jeth1, — jeth2, - - - jeth3 and - - jeth4.

\section{Pressure spectra}

In order to assess the validity of the simulations, the LES acoustic spectra are first compared to those recorded in the experiments at the MARTEL test bench. The sound pressure levels obtained at $z=-1.3 r_{0}$ and $r=10.5 r_{0}$ for jeth1 and jeth2 are represented in figure 6 , along with the experimental data. For both jets, a fair agreement is found. The 
spectra are broadband and exhibit bumps for Strouhal numbers between 0.02 and 0.4. For jeth1, in figure 6a), the bump frequencies from the simulations and the experiments do not agree very well. However, the difference in SPL does not exceed $1 \mathrm{~dB}$ between $S t=0.04$ and 0.2. For jeth2, in figure 6 (b), the discrepancies between the simulations and the experiments are stronger. In particular, the main peak observed at a similar frequency $S t \approx 0.07$ is $3 \mathrm{~dB}$ higher in the LES than in the experiments. The frequency of this peak can be compared with the central frequency $f_{p}$ of BBSAN estimated by the model of Harper-Bourne \& Fisher [34]

$$
f_{p}=\frac{u_{c}}{L_{s}\left(1-M_{c} \cos \theta\right)}
$$

where $M_{c}=u_{c} / c_{0}$ is the convection Mach number and $\theta$ is the angle between the jet direction and the far-field observation point. For $\theta=180^{\circ}$, a Strouhal number of $S t_{p}=0.0635$ is obtained using equation (4), which is close to the frequency $S t=0.065$ of the peak in the LES spectrum. Therefore, the peak in the spectra appears to be related to the BBSAN. Its higher amplitude in the simulation may be due to the state of the mixing layer at the nozzle exit. Indeed, the turbulent intensity at the nozzle exit is equal to $1.5 \%$ in the present simulations, but is most probably higher in the experiments, which can affect the sound radiated by the jet [35].

(a)

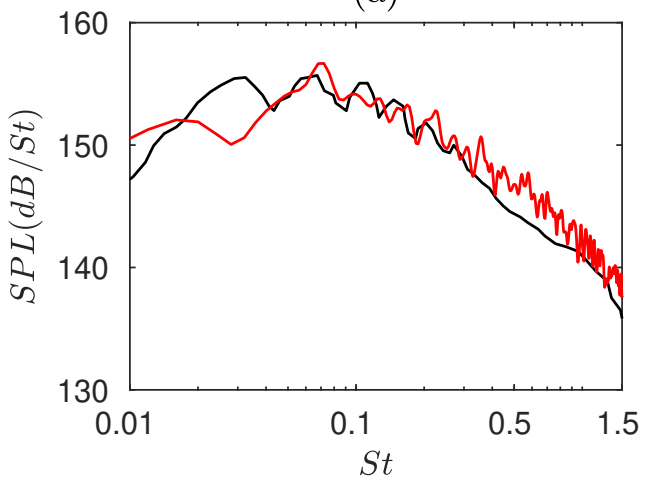

(b)

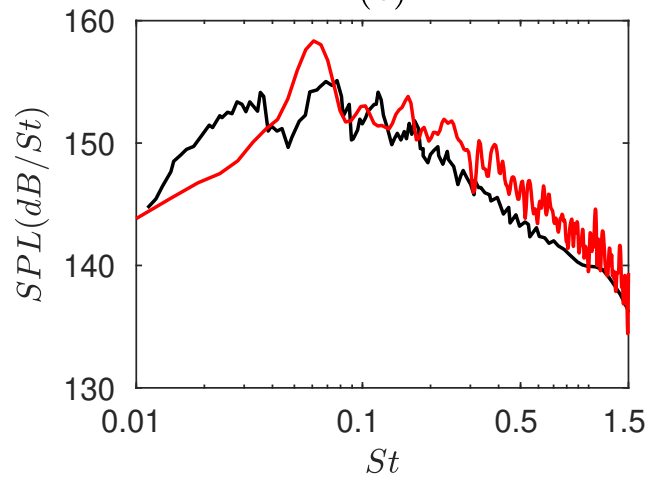

Fig. 6 Sound pressure levels at $z=-1.3 r_{0}$ and $r=10.5 r_{0}$ for (a) jeth1 and (b) jeth2; - experiments, — present simulations.

The acoustic spectra estimated at three locations, namely near the nozzle, near the plate and downstream of the plate, are displayed in figure 7 The pressure spectra calculated at $z=0$ and $r=2 r_{0}$ near the nozzle are shown in figure 7 (a). For the free jet, a peak centered on $S t=0.06$ appears. As discussed above, this peak is attributed to the BBSAN. For jeth0, the levels are approximately $12 \mathrm{~dB}$ higher, and the strongest components are found around $S t=0.04$. They may be generated by a feedback loop establishing between the nozzle and the plate. However, the absence of strongly emerging tones implies that such a resonance is weak. For the plates with a hole, the pressure levels decrease as the hole diameter increases. This suggests that the interactions between the jet and the hole edges are weaker for larger holes, leading to a diminution of the impingement noise. For jeth1 and jeth2, compared with the no-hole case, the acoustic levels are reduced for $S t \geq 0.1$, by about $3 \mathrm{~dB}$ for jeth 1 and $4 \mathrm{~dB}$ for jeth2. For jeth3, a noise reduction of $4 \mathrm{~dB}$ is observed for all frequencies with respect to jeth2. Finally, for jeth 4 , for $S t \leq 0.2$, the pressure levels decrease by approximately $2 \mathrm{~dB}$ relative to jeth 3 .

The acoustic spectra computed at $z=20 r_{0}$ and $r=15 r_{0}$, in the direction of propagation of the Mach waves, are represented in figure 8 b). For all jets, the spectra display a similar shape, reaching a peak at a Strouhal number between 0.11 and 0.15 . They are almost superimposed for $S t>0.5$. At lower frequencies, the levels are highest for jeth0, they decrease as the hole is larger and they are lowest for the free jet. In comparison to those for the free jet, for $S t<0.2$, they increase by at maximum $14 \mathrm{~dB}$ for jeth $0,12 \mathrm{~dB}$ for jeth 1 and jeth 2 , and $10 \mathrm{~dB}$ for jeth 3 and jeth 4 . The jet impingement on the plate therefore seems to produce stronger acoustic waves of low frequencies.

The sound pressure levels obtained downstream of the plate, at $z=40 r_{0}$ and $r=15 r_{0}$, are plotted in figure $8(\mathrm{c})$. For all jets, the spectra are centered on a peak at Strouhal numbers around $S t \approx 0.09$. The acoustic levels are similar for $S t \geq 0.5$. For lower Strouhal numbers, they are minimal for jeth1 and increase with the hole diameter. Compared with those for jeth1, the levels increase by roughly $2 \mathrm{~dB}$ for jeth $2,3 \mathrm{~dB}$ for jeth $3,4 \mathrm{~dB}$ for jeth 4 and $5 \mathrm{~dB}$ for the free jet. 
Indeed, for the impinging jets, the Mach waves generated in the jet shear layers are shielded by the plate. A significant part of the flow is also diverted in the wall jet, leading to a weaker noise radiation of the jets downstream of the plate.

(a)

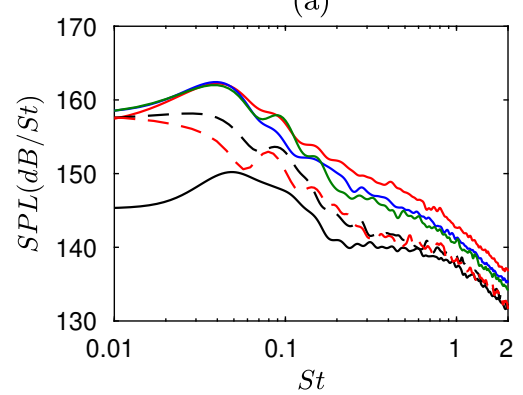

(b)

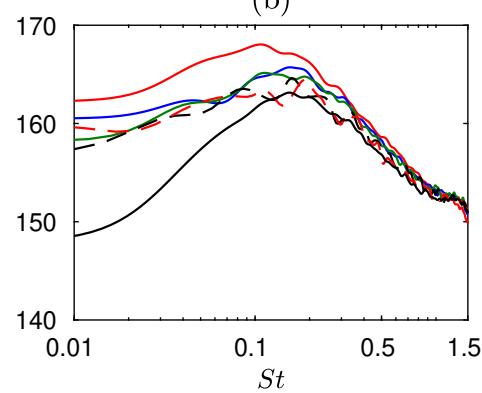

(c)

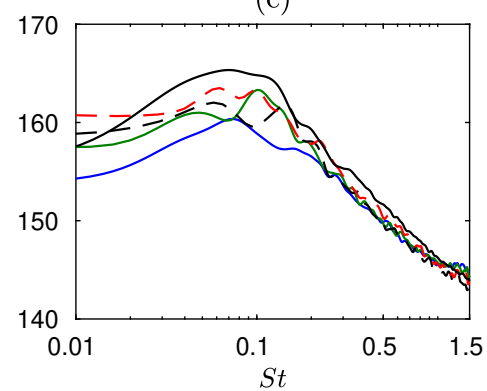

Fig. 7 Sound pressure levels (SPL) at (a) $z=0$ and $r=2 r_{0}$, (b) $z=20 r_{0}$ and $r=15 r_{0}$ and (c) $z=40 r_{0}$ and $r=15 r_{0}$ as a function of the Strouhal number $S t:-$ jeth0, - jeth1, - jeth2, - - jeth3, - - jeth4 and - jetfree.

In order to highlight the azimuthal structure of the jet acoustic fields, the contributions of the first four azimuthal modes to the pressure spectra at $z=0$ and $r=2 r_{0}$ are shown in figure 8 . In all cases, the axisymmetric mode $n_{\theta}=0$ is predominant at Strouhal numbers lower than 0.1, for which the acoustic levels are the highest. For $S t \geq 0.2$, the contributions of the axisymmetric mode weaken significantly. For higher azimuthal modes, in all cases, the levels are negligible at Strouhal numbers lower than 0.1, then they increase with the frequency to reach those for the axisymmetric mode. Therefore, the jet impingement on the plate does not change the azimuthal structure of the upstream acoustic field. Some differences can be however noticed between the spectra of the free and those of the impinging jets. For the impinging jets in figures $8(\mathrm{a}-\mathrm{d})$, a bump is observed at $S t=0.04$ for $n_{\theta}=0$. Secondary humps around $S t \approx 0.08$ are also noted for jeth2, jeth3 and jeth4. Such humps are not found for the free jet. They may be related to feedback loops establishing between the nozzle and the plate.

(a)

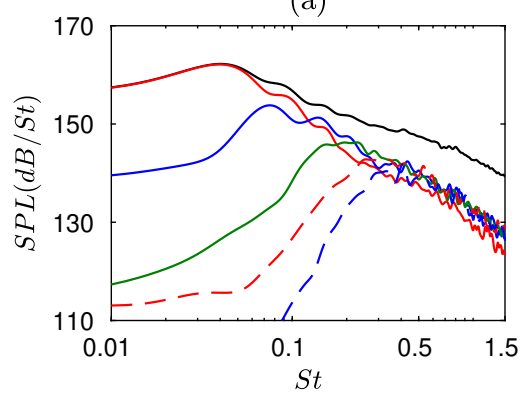

(d)

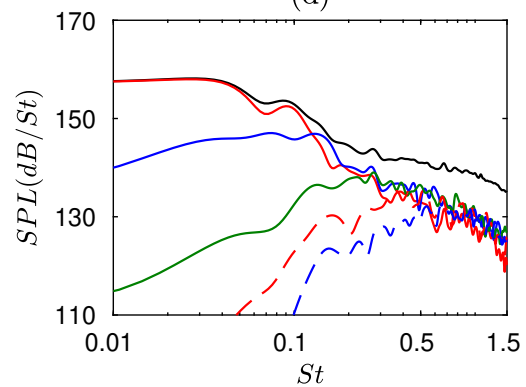

(b)

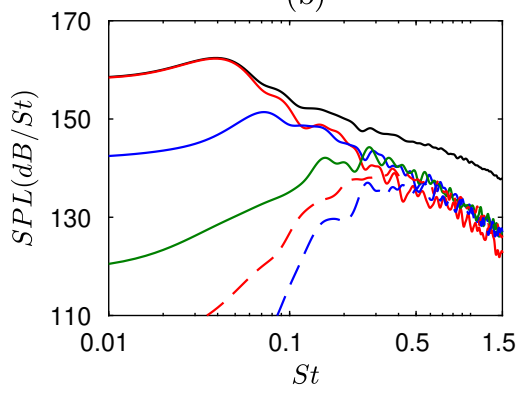

(e)

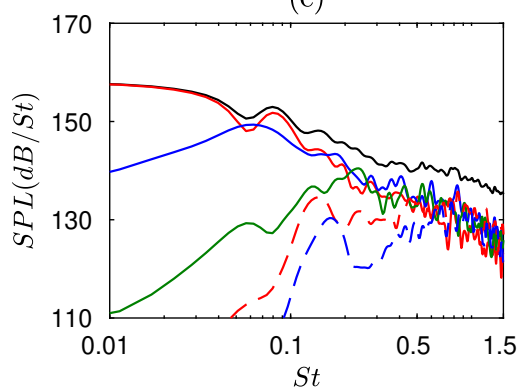

(c)

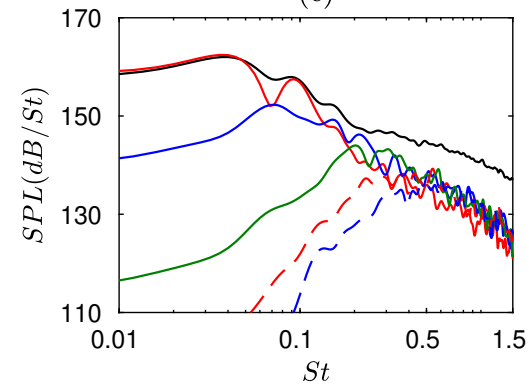

(f)

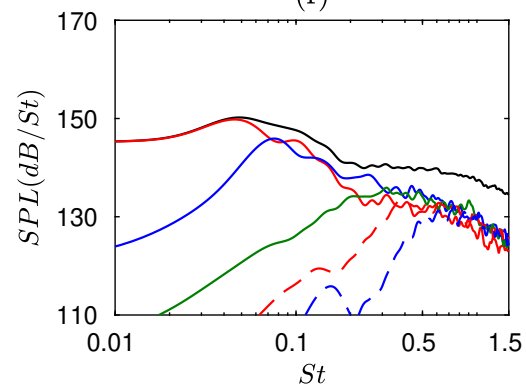

Fig. 8 Sound pressure spectra at $z=0$ and $r=2 r_{0}$ for (a) jeth0, (b) jeth1, (c) jeth2, (d) jeth3, (e) jeth4 and (f) jetfree: - full spectra, and for modes $-n_{\theta}=0,-n_{\theta}=1,-n_{\theta}=2,--n_{\theta}=3$ and $--n_{\theta}=4$. 


\section{E. Spatial Fourier decomposition of the pressure fields}

A two-dimensional spatial Fourier transform in the radial and axial directions has been applied to the pressure fields of the jets using the method developed by Nonomura et al. [36] for free jets at an exit Mach number of 2. For the jets impinging on a perforated plate, the results are similar and therefore only those for jeth 1 are presented. The regions where this Fourier transform is performed are represented in figure 9 for jetfree, jeth 0 and jeth 1 . In the three cases, they extend from $z=5 r_{0}$ down to $z=25 r_{0}$ axially and from $r=5 r_{0}$ out to $r=15 r_{0}$ radially. These areas are chosen far enough from the jet so that the fluctuations of pressure are only of acoustic nature. In all cases, Mach waves are seen to propagate downstream. For jetfree, in figure 9 (a), upstream-propagating waves of very weak amplitude are also observed for $z \leq 10 r_{0}$. For jeth 0 and jeth1, in figures 9 (b-c), strong pressure waves originating from the plate propagate upstream. Their amplitude is lower for the plate with a hole.

(a)

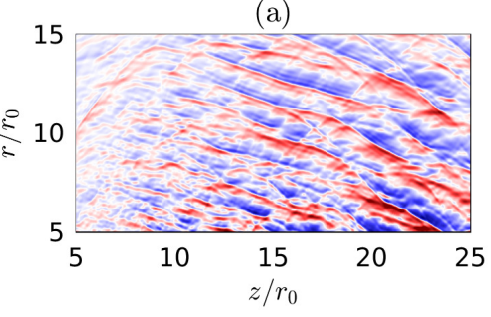

(b)

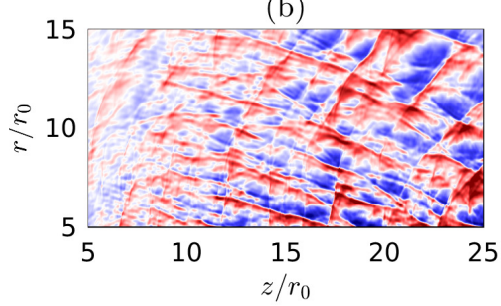

(c)

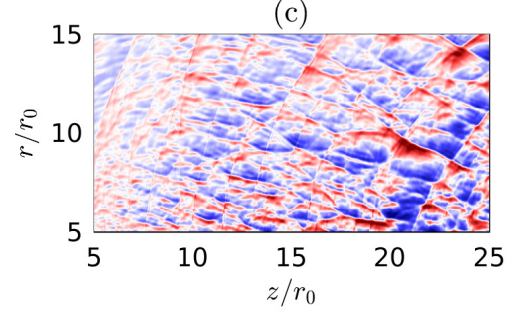

Fig. 9 Pressure fields where the two-dimensional spatial Fourier transform is applied for (a) jetfree, (b) jeth0 and (c) jeth1. The color scale level ranges from $\pm 0.1 p_{0}$, from blue to red.

The spatial Fourier decomposition is performed at each recorded time. The results are then time-averaged. The amplitudes thus obtained are presented in figure 10 as a function of the radial and axial wavenumbers $k_{r}$ and $k_{z}$. For the free jet in figure 10(a), lobes are observed in the quadrants where $k_{r}$ and $k_{z}$ have the same sign, indicating that they are associated with downstream-propagating waves. The lobes are aligned with the direction of propagation of the Mach waves, showing that the latter are the main acoustic components in the pressure field. Similar lobes can be seen for the impinging jets in figures $10(\mathrm{~b}, \mathrm{c})$. However, lobes are also found in quadrants with $k_{r}$ and $k_{z}$ of opposite signs, indicating the presence of upstream-propagating waves. The orientation of the lobes is compared with the direction of propagation of reflected Mach waves, assuming a specular reflection on the plate. The orientation of the lobes does not agree with this direction, implying that the reflections of Mach waves have negligible contributions in the acoustic region considered. The amplitude fields also provide information on the frequency content of the sound waves, since in the wavenumber plane $\left(k_{z}, k_{r}\right)$, the distance of a point to the origin is proportional to the frequency. For the impinging jets in figures $10(\mathrm{~b}, \mathrm{c})$, the lobes related to upstream-propagating waves do not extend as far from the origin as those of the downstream-propagating waves, indicating that the frequencies of the upstream noise components are lower than those of the downstream noise components. This result is consistent with the sound spectra of figure 7 This provides further evidence that the main upstream noise components are not generated by the reflections of the downstream noise on the plate. Moreover, the levels in the quadrants with $k_{z}$ and $k_{r}$ of opposite signs are lower for jeth1 than for jeth0, supporting the idea that the noise in the upstream direction is mainly produced by the impingement of turbulent structures on the plate.

\section{F. Two-dimensional spatial correlations}

In order to visualize the different noise components, two-dimensional spatial correlations of the jet pressure fields have been calculated in a section $(z, r)$. The fluctuating pressure $p^{\prime}$ at a reference point $\left(z_{1}, r_{1}\right)$ at time $t$ is correlated with the pressure fluctuations in the plane $(z, r)$ at time $t+\delta t$, giving the dimensionless coefficient $\mathcal{R}$

$$
\mathcal{R}(r, z, \delta t)=\frac{\left\langle p^{\prime}\left(r_{1}, z_{1}, t\right) p^{\prime}(r, z, t+\delta t)\right\rangle}{\left\langle p^{\prime 2}\left(r_{1}, z_{1}, t\right)\right\rangle^{1 / 2}\left\langle p^{\prime 2}(r, z, t)\right\rangle^{1 / 2}}
$$

where $\delta t$ is the time delay between the signals and $\langle$.$\rangle denotes time averaging. In this manner, the shapes and the time$ variations of the waves correlated with the pressure fluctuations at the reference point are revealed. The correlation coefficient $\mathcal{R}$ has been evaluated for all jets at different reference points to study the sound radiation upstream and downstream of the plate. The results for the impinging jets are similar. Therefore, only those for jeth 2 are shown. 

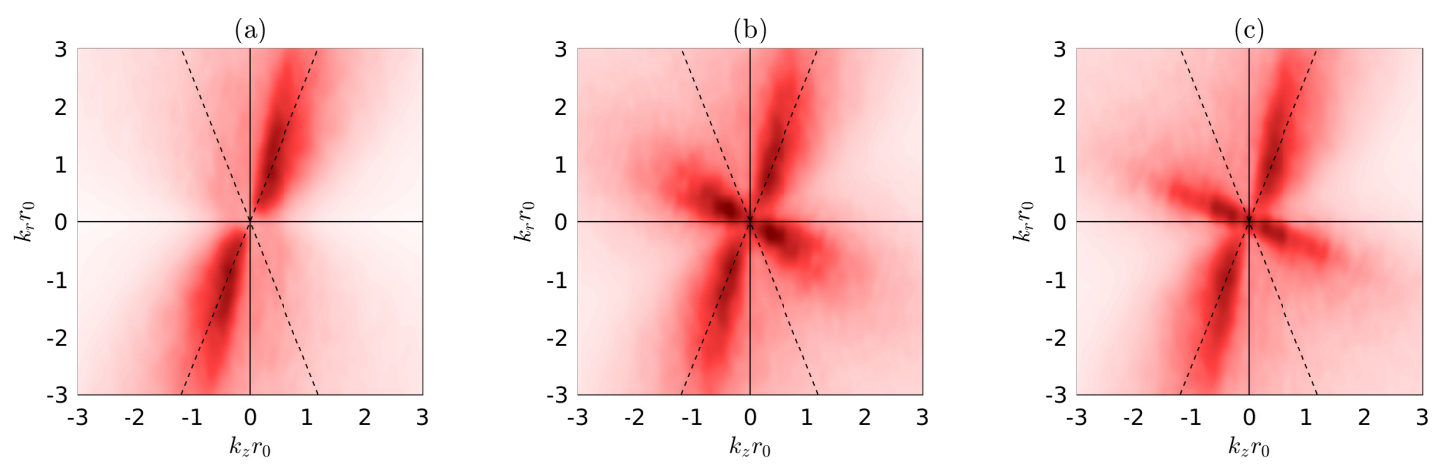

Fig. 10 Two-dimensional wavenumber spectra as a function of $\left(k_{r}, k_{z}\right)$ for (a) jetfree, (b) jeth0 and (c) jeth1; - - - propagation directions of $\alpha=6^{\circ}$ and $112^{\circ}$ of the incident and reflected Mach waves. The color scale levels spread over $35 \mathrm{~dB}$ from white to red.

\section{Upstream of the plate}

The correlations are first calculated for jeth2 for a reference point near the nozzle at $z_{1}=0$ and $r_{1}=2 r_{0}$, in order to highlight the upstream acoustic radiation. The results obtained are presented in figure 11 for time delays $\delta t=-100 r_{0} / u_{e}$, $-50 r_{0} / u_{e}$ and 0 . For $\delta t=-100 r_{0} / u_{e}$, in figure 11(a), three circular fronts of positive correlations are observed, located around $z=0, z=10 r_{0}$ and $z=20 r_{0}$ for $r \approx 0$. They are separated by a distance close to the wavelength $\lambda=10.2 r_{0}$ corresponding to the Strouhal number $S t=0.04$ of the strongest noise components near the nozzle. The levels of correlations are highest for the front at $z \approx 20 r_{0}$. The latter is aligned with a circle centered on a point at $z=L$ and $r=6 r_{0}$, suggesting that the main upstream noise components are generated on the plate. For $\delta t=-50 r_{0} / u_{e}$, in figure 11 b), the correlation levels are higher than previously. The wavefronts propagate to the reference point over a distance of about $10 r_{0}$, which is consistent with the distance $c_{0} \times 50 r_{0} / u_{e}$ travelled by a sound wave during $50 r_{0} / u_{e}$. For $\delta t=0$, in figure 11 (c), correlation is equal to 1 on the reference point, as expected. The wavefront is circular, as for the previous time delays, and strong levels are visible inside and outside of the jet flow in the vicinity of the nozzle exit.
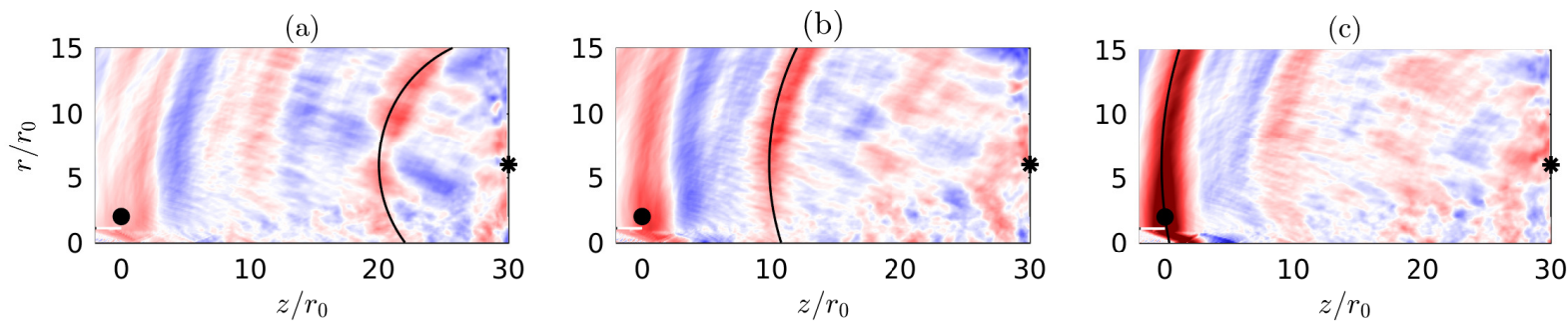

Fig. 11 Correlations $\mathcal{R}$ of $p^{\prime}\left(r=2 r_{0}, z=0, t\right)$ with $p^{\prime}(r, z, t+\delta t)$ for (a) $\delta t=-100 u_{e} / r_{0},(b) \delta t=-50 r_{0} / u_{e}$ and (c) $\delta t=0$ for jeth2; - circle centered on $*$ at $\left(z=L, r=6 r_{0}\right)$. The color scale ranges between \pm 0.5 , from blue to red.

The correlation levels are then computed for jeth2 for a reference point near the plate at $z=20 r_{0}$ and $r=15 r_{0}$, with the aim of examining the acoustic waves propagating in the radial direction. The correlations thus computed are shown in figure 12 for $\delta t=-40 r_{0} / u_{e},-20 r_{0} / u_{e}$ and 0 . For $\delta t=-40 r_{0} / u_{e}$, in figure 12(a), two areas of significant correlations appear. The first one is aligned with the direction $\beta=158^{\circ}$ of the incident Mach waves observed in the pressure snapshots of figure 3 The second one has the shape of a circular arc and is aligned with a circle centered on a point at $z=L$ and $r=6 r_{0}$ on the plate. In particular, no correlation front parallel to possible reflected Mach waves can be seen, indicating that the contributions of these reflections to the radiated sound are negligible at the reference point. Therefore, the acoustic radiation consists of two main components, namely the incident Mach waves and the impingement noise. For $\delta t=-20 r_{0} / u_{e}$, in figure $12 \mathrm{~b}$ ), these two contributions propagate to the reference point. Finally, for $\delta t=0$, in figure $12(\mathrm{c})$, they reach that point, causing a correlation level of 1 . 
(a)

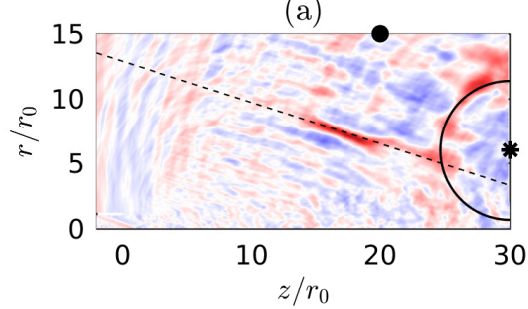

(b)

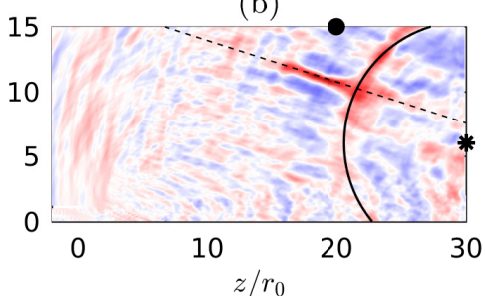

(c)

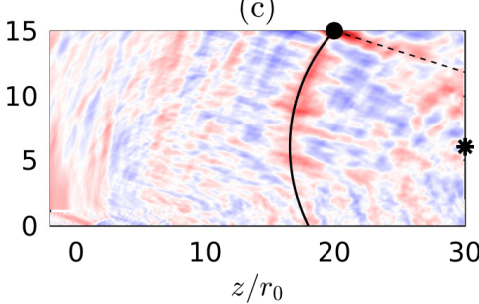

Fig. 12 Correlations $\mathcal{R}$ of $p^{\prime}\left(r=15 r_{0}, z=20 r_{0}, t\right)$ with $p^{\prime}(r, z, t+\delta t)$ for $(a) \delta t=-40 r_{0} / u_{e},(b) \delta t=-20 r_{0} / u_{e}$ and (c) $\delta t=0$ for jeth2; - circle centered on $*$ at $\left(z=L, r=6 r_{0}\right)$ and $--\operatorname{direction} \beta=158^{\circ}$ with respect to the jet axis. The color scale ranges between \pm 0.5 , from blue to red.

\section{Downstream of the plate}

Two-dimensional spatial correlations are estimated for a reference point at $z=40 r_{0}$ and $r=15 r_{0}$ for the free jet, in order to visualize the noise radiation in the downstream direction. The correlation coefficients obtained are given in figures 13 for the time delays $\delta t=-75 r_{0} / u_{e},-45 r_{0} / u_{e}$ and $-15 r_{0} / u_{e}$. For $\delta t=-75 r_{0} / u_{e}$, in figure 13(a), a spot of strong correlations is found in the jet flow at $z=30 r_{0}$. An inclined front of high positive correlations also emerges from the flow at this position. These features indicate the generation of Mach waves by flow structures convected at a supersonic speed. Similar results were found in previous studies for temporal jets at Mach numbers of 2 and 3 [28, 37]. Later, for $\delta t=-45 r_{0} / u_{e}$, in figure 13 ,b), the pressure wave leaves the flow, creating a straight band of strong correlations at an angle of $\beta=148^{\circ}$ with respect to the jet axis. This angle is lower than that of the Mach waves observed for $z<30 r_{0}$, which can be explained by the decrease of the convection velocity of the jet vortical structures with the axial distance. Finally, for $\delta t=-15 r_{0} / u_{e}$, in figure 13 (c), the wave propagates to the reference point.
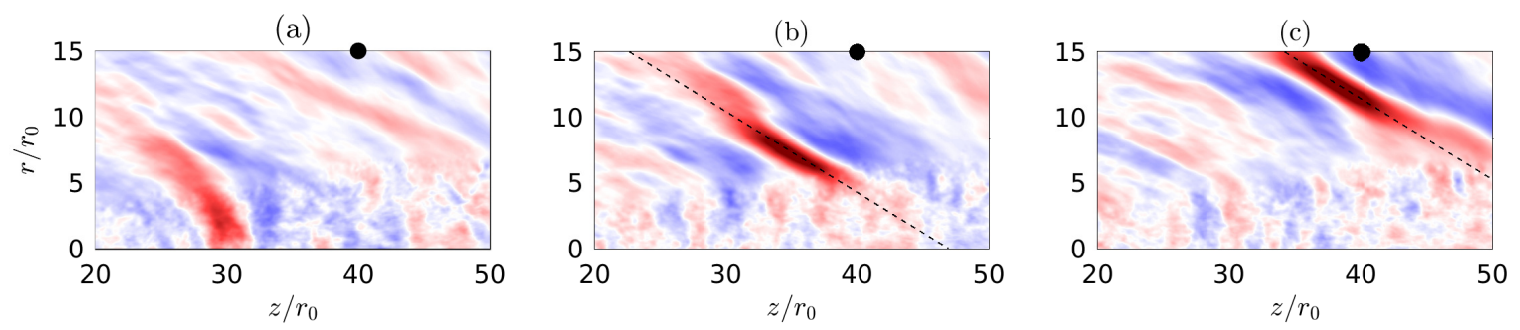

Fig. 13 Correlations $\mathcal{R}$ of $p^{\prime}\left(r=15 r_{0}, z=40 r_{0}, t\right)$ with $p^{\prime}(r, z, t+\delta t)$ for $(a) \delta t=-75 r_{0} / u_{e},(b) \delta t=-45 r_{0} / u_{e}$ and (c) $\delta t=-15 r_{0} / u_{e}$ for jetfree. The dashed line indicates the direction $\beta=148^{\circ}$ with respect to the jet axis. The color scale ranges between $\mathbf{\pm 0 . 5}$, from blue to red.

Correlations are finally evaluated for the same reference point for jeth2 to give insight into the acoustic radiation downstream of the plate. The results obtained for the same time delays as for the free jet are presented in figure 14 . For $\delta t=-75 r_{0} / u_{e}$, in figure $14(\mathrm{a})$, significant correlation levels are noted near the hole in the plate, revealing interactions between the jet flow and the plate. Upstream of the plate, no clear organization of the correlations field is observed, whereas downstream of the plate, fronts of positive correlations centered on the hole are seen. For $\delta t=-45 r_{0} / u_{e}$, in figure 14 (b), a front of strong correlations is noticed downstream of the plate. In comparison with the wavefront observed at the same time delay for the corresponding free jet, in figure 13 (b), it is more curved and is not aligned with the angle $\beta=148^{\circ}$. These differences in shape indicate that the sound waves downstream of the plate for jeth2 are not produced by Mach waves, but that they result from the interactions between the jet turbulent structures and the plate visible at the previous time delay. For $\delta t=-15 r_{0} / u_{e}$, in figure 14(c), as for the free jet, the wave travels to the reference point.

\section{Conclusion}

In this paper, compressible large-eddy simulations have been used to examine the sound radiation of overexpanded jets at an exhaust Mach number of 3.1 impinging on a plate with and without a hole for different hole diameters $h$. The effects of the plate and of the hole on the flow and acoustic fields have been investigated by comparing the fields 

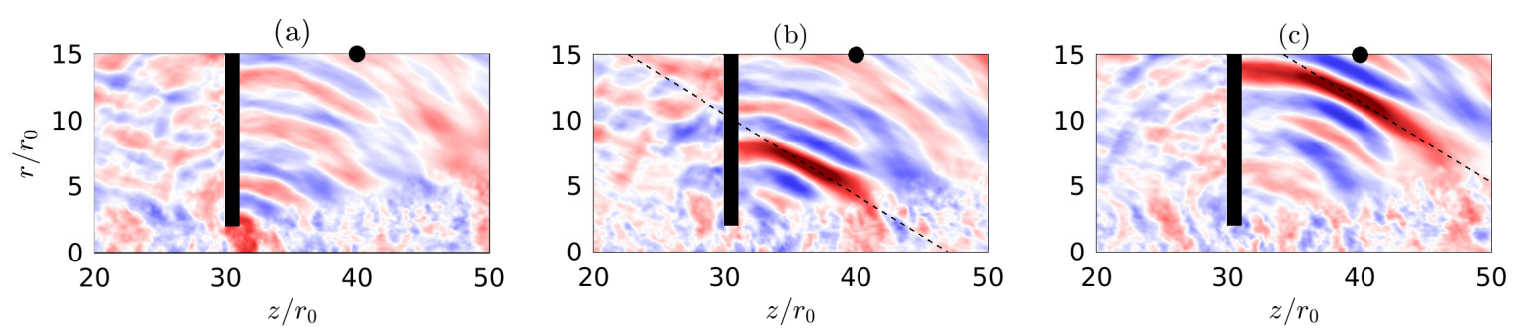

Fig. 14 Correlations $\mathcal{R}$ of $p^{\prime}\left(r=15 r_{0}, z=40 r_{0}, t\right)$ with $p^{\prime}(r, z, t+\delta t)$ for $(a) \delta t=-75 r_{0} / u_{e},(b) \delta t=-45 r_{0} / u_{e}$ and (c) $\delta t=-15 r_{0} / u_{e}$ for jeth2. The dashed line indicates the direction $\beta=148^{\circ}$ with respect to the jet axis. The color scale ranges between $\pm \mathbf{0 . 5}$, from blue to red.

obtained to those of the corresponding free jet. For all impinging jets, two-dimensional spatial correlations and spatial Fourier transforms reveal that the upstream acoustic radiation is mainly produced by the impingement of the turbulent structures of the jet flow on the plate, and that the sound reflections on the plate have negligible contributions near the jet nozzle. The upstream sound radiation is weaker as the hole diameter is larger. Compared with the full-plate case, for $h \leq 2 D$, the near-nozzle sound pressure levels are reduced at high frequencies only, while for $h \geq 3 D$, they are lower for all frequencies. The latter reduction over the whole frequency range is due to weaker interactions between the turbulent structures of the jets and the plate. Indeed, for $h \leq 2 D$, the jet mixing layers impinge on the plate and are strongly distorted by the impingement, whereas for $h \geq 3 D$, they pass through the hole and are only slightly distorted. These interactions are especially weak for the largest hole $h=4 D$, resulting in a decrease by $4 \mathrm{~dB}$ of the overall acoustic level in the vicinity of the nozzle in comparison with the full plate. Moreover, for $h \leq 3 D$, a large peak is found at a low frequency in the near-nozzle pressure spectra. This peak is too wide and its amplitude is too low to clearly conclude about the existence of a resonance phenomenon in the present cases. In further investigations, rocket jets impinging on plates at a smaller nozzle-to-plate distance, typically $L=15 r_{0}$, could be considered in order to investigate the possible establishment of feedback phenomena and the generation of acoustic tones for high Mach number jets.

\section{Acknowledgments}

This work was financed by ArianeGroup and the DGA (Direction Générale de l'Armement). It was granted access to the HPC resources of PMCS2I (Pôle de Modélisation et de Calcul en Sciences de l'Ingénieur et de l'Information) of Ecole Centrale de Lyon, PSMN (Pôle Scientifique de Modélisation Numérique) of ENS de Lyon, members of FLMSN (Fédération Lyonnaise de Modélisation et Sciences Numériques), partner of EQUIPEX EQUIP@MESO, and to the resources of IDRIS (Institut du Développement et des Ressources en Informatique Scientifique) under the allocation 2020-2a0204 made by GENCI (Grand Equipement National de Calcul Intensif). It was performed within the framework of the LABEX CeLyA (ANR-10-LABX-0060) of Université de Lyon, within the program Investissements d'Avenir (ANR-16-IDEX-0005) operated by the French National Research Agency (ANR).

\section{References}

[1] Kawai, S., Tsutsumi, S., Takaki, R., and Fujii, K., "Computational aeroacoustic analysis of overexpanded supersonic jet impingement on a flat plate with/without hole," ASME/JSME 2007 5th Joint Fluids Engineering Conference, American Society of Mechanical Engineers, 2007, pp. 1163-1167. https://doi.org/10.1115/FEDSM2007-37563

[2] Tsutsumi, S., Takaki, R., Ikaida, H., and Terashima, K., "Numerical Aeroacoustics analysis of a scaled solid jet impinging on flat plate with exhaust hole," 30th International Symposium on Space Technology and Science, 2015.

[3] Troyes, J. N., Vuillot, F., Langenais, A., and Lambaré, H., "Coupled CFD-CAA Simulation of the Noise Generated by a Hot Supersonic Jet Impinging on a Flat Plate with Exhaust Hole,” AIAA Paper 2019-2752, 2019. https://doi.org/10.2514/6.2019-2752

[4] Tsutsumi, S., Takaki, R., Shima, E., Fujii, K., and Arita, M., "Generation and Propagation of Pressure Waves from H-IIA Launch Vehicle at Lift-off," AIAA Paper 2008-390, 2008. https://doi.org/10.2514/6.2008-390.

[5] Gojon, R., Bogey, C., and Marsden, O., "Investigation of tone generation in ideally expanded supersonic planar impinging jets using large-eddy simulation,” J. Fluid Mech., Vol. 808, 2016, pp. 90-115. https://doi.org/10.1017/jfm.2016.628 
[6] Henderson, B., Bridges, J., and Wernet, M., "An experimental study of the oscillatory flow structure of tone-producing supersonic impinging jets,” J. Fluid Mech., Vol. 542, 2005, pp. 115-137. https://doi.org/10.1017/S0022112005006385.

[7] Umeda, Y., and Ishii, R., "Hole tone generation from highly choked jets," J. Acoust. Soc. Am., Vol. 94, No. 2, 1993, pp. 1058-1066. https://doi.org/10.1121/1.406952

[8] Varé, M., and Bogey, C., "Large-eddy simulations of round jets at a Mach number of 0.9 impinging on a plate with and without a hole," AIAA Paper 2020-2549, 2020. https://doi.org/10.2514/6.2020-2549.

[9] Piantanida, S., and Berterretche, P., "Acoustic characterization of two parallel supersonic jets," INTER-NOISE and NOISE-CON Congress and Conference Proceedings, Vol. 259, Institute of Noise Control Engineering, 2019, pp. 4251-4262.

[10] Doty, M., and McLaughlin, D., "Acoustic and mean flow measurements of high-speed, helium-air mixture jets," Intl J. of Aeroacoust., Vol. 2, No. 3, 2003, pp. 293-333. https://doi.org/10.1260/147547203322986151

[11] Bogey, C., Marsden, O., and Bailly, C., "Large-eddy simulation of the flow and acoustic fields of a Reynolds number $10^{5}$ subsonic jet with tripped exit boundary layers," Phys. Fluids, Vol. 23, No. 3, 2011, p. 035104. https://doi.org/10.1063/1.3555634

[12] Liu, J., and Khine, Y.-Y., "Nozzle boundary-layer separation near the nozzle exit in highly overexpanded jets," AIAA Paper 2020-2561, 2020. https://doi.org/10.2514/6.2020-2561

[13] Bogey, C., and Sabatini, R., "Effects of nozzle-exit boundary-layer profile on the initial shear-layer instability, flow field and noise of subsonic jets," J. Fluid Mech., Vol. 876, 2019, pp. 288-325. https://doi.org/10.1017/jfm.2019.546

[14] Bogey, C., "Acoustic tones in the near-nozzle region of jets: characteristics and variations between Mach numbers 0.5 and 2 ," $J$. Fluid Mech., 2021. https://doi.org/10.1017/jfm.2021.426

[15] Varé, M., and Bogey, C., "Generation of acoustic tones in round jets at a Mach number of 0.9 impinging on a plate with and without a hole," submitted to J. Fluid Mech., 2021.

[16] Bogey, C., and Bailly, C., "A family of low dispersive and low dissipative explicit schemes for flow and noise computations," $J$. Computat. Phys., Vol. 194, No. 1, 2004, pp. 194-214. https://doi.org/10.1016/j.jcp.2003.09.003

[17] Bogey, C., De Cacqueray, N., and Bailly, C., "A shock-capturing methodology based on adaptative spatial filtering for high-order non-linear computations," J. Comput. Phys., Vol. 228, No. 5, 2009, pp. 1447-1465. https://doi.org/10.1016/j.jcp.2008.10.042

[18] Fauconnier, D., Bogey, C., and Dick, E., “On the performance of relaxation filtering for large-eddy simulation,” J. Turbul., Vol. 14, No. 1, 2013, pp. 22-49. https://doi.org/10.1080/14685248.2012.740567

[19] Tam, C., and Dong, Z., "Radiation and outflow boundary conditions for direct computation of acoustic and flow disturbances in a non uniform mean flow," J. Comput. Acoust., Vol. 4, No. 02, 1996, pp. 175-201. https://doi.org/10.1142/S0218396X96000040

[20] Bogey, C., and Bailly, C., "Three-dimensional non-reflective boundary conditions for acoustic simulations: far-field formulation and validation test cases," Acta Acustica united with Acustica, Vol. 88, No. 4, 2002, pp. 463-471.

[21] Mohseni, K., and Colonius, T., "Numerical treatment of polar coordinate singularities," J. Comput. Phys., Vol. 157, No. 2, 2000, pp. 787-795. https://doi.org/10.1006/jcph.1999.6382

[22] Bogey, C., De Cacqueray, N., and Bailly, C., "Finite differences for coarse azimuthal discretization and for reduction of effective resolution near origin of cylindrical flow equations," J. Comput. Phys., Vol. 230, No. 4, 2011, pp. 1134-1146. https://doi.org/10.1016/j.jcp.2010.10.031

[23] Pineau, P., and Bogey, C., "Study of the generation of shock waves by high-speed jets using conditional averaging," AIAA Paper 2018-3305, 2018. https://doi.org/10.2514/6.2018-3305

[24] Pineau, P., and Bogey, C., "Links Between Steepened Mach Waves and Coherent Structures for a Supersonic Jet," AIAA J., Vol. 59, No. 5, 2021, pp. 1673-1681. https://doi.org/10.2514/1.J059859

[25] De Cacqueray, N., Bogey, C., and Bailly, C., "Investigation of a high-Mach-number overexpanded jet using large-eddy simulation," AIAA J., Vol. 49, No. 10, 2011, pp. 2171-2182. https://doi.org/10.2514/1.J050952

[26] Langenais, A., Vuillot, F., Troyes, J., and Bailly, C., "Accurate simulation of the noise generated by a hot supersonic jet including turbulence tripping and nonlinear acoustic propagation," Phys. Fluids, Vol. 31, No. 1, 2019 , p. 016105. https://doi.org/10.1063/1.5050905. 
[27] Nonomura, T., and Fujii, K., "Overexpansion effects on characteristics of Mach waves from a supersonic cold jet," AIAA J., Vol. 49, No. 10, 2011, pp. 2282-2294. https://doi.org/10.2514/1.J051054

[28] Pineau, P., and Bogey, C., "Steepened Mach waves near supersonic jets: study of azimuthal structure and generation process using conditional averages," J. Fluid Mech., Vol. 880, 2019, pp. 594-619. https://doi.org/10.1017/jfm.2019.729

[29] Troyes, J., Vuillot, F., Lambaré, H., and Espinosa Ramos, A., "Numerical study of free supersonic hot jet on unstructured grids with emphasis on aerodynamics and resulting radiated noise," AIAA Paper 2016-2734, 2016. https://doi.org/10.2514/6.2016-2734

[30] Tam, C., and Tanna, H., "Shock associated noise of supersonic jets from convergent-divergent nozzles," J. Sound Vib., Vol. 81, No. 3, 1982, pp. 337-358. https://doi.org/10.1016/0022-460X(82)90244-9

[31] Pack, D., "A note on Prandtl's formula for the wave-length of a supersonic gas jet," The Quarterly Journal of Mechanics and Applied Mathematics, Vol. 3, No. 2, 1950, pp. 173-181. https://doi.org/10.1093/qjmam/3.2.173

[32] Tam, C., Jackson, J., and Seiner, J., "A multiple-scales model of the shock-cell structure of imperfectly expanded supersonic jets,” J. Fluid Mech., Vol. 153, 1985, pp. 123-149. https://doi.org/10.1017/S0022112085001173

[33] Eggers, J., "Velocity profiles and eddy viscosity distributions downstream of a Mach 2.22 nozzle exhausting to quiescent air," NASA TN D-3601, 1966.

[34] Harper-Bourne, M., “The noise from shock waves in supersonic jets-Noise Mechanism,” Agard Cp-131, 1974.

[35] Bogey, C., Marsden, O., and Bailly, C., "Influence of initial turbulence level on the flow and sound fields of a subsonic jet at a diameter-based Reynolds number of 105," J. Fluid Mech., Vol. 701, 2012, pp. 352-385. https://doi.org/10.1017/jfm.2012.162

[36] Nonomura, T., Nakano, H., Ozawa, Y., Terakado, D., Yamamoto, M., Fujii, K., and Oyama, A., "Large eddy simulation of acoustic waves generated from a hot supersonic jet," Shock Waves, Vol. 29, 2019, p. 1133-1154. https://doi.org/10.1007/s00193019-00895-2

[37] Bogey, C., and Pineau, P., "Potential-core closing of temporally developing jets at Mach numbers between 0.3 and 2 : Scaling and conditional averaging of flow and sound fields," Phys. Rev. Fluids, Vol. 4, No. 12, 2019, p. 124601. https://doi.org/10.1103/PhysRevFluids.4.124601 